\title{
A Case of Shoulder Desmoid-Type Fibromatosis Missed on an Initial Work Up: Case Report and Literature Review
}

\author{
Michael Byrd ${ }^{*}$, William Barfield², Emily Darr ${ }^{2}$ \\ ${ }^{1}$ College of Medicine, Medical University of South Carolina, Charleston, SC, USA; ${ }^{2}$ Department of Orthopaedics \& \\ Physical Medicine, Medical University of South Carolina, Charleston, SC, USA
}

Correspondence to: Michael Byrd, byrdm@musc.edu

Keywords: Desmoid-Type Fibromatosis, All Rehabilitation, All Pain

Received: September 30, $2020 \quad$ Accepted: October 27, $2020 \quad$ Published: October 30, 2020

Copyright $\odot 2020$ by author(s) and Scientific Research Publishing Inc.

This work is licensed under the Creative Commons Attribution International License (CC BY 4.0).

http://creativecommons.org/licenses/by/4.0/

\section{(c) (i) Open Access}

\section{ABSTRACT}

Desmoid-type fibromatosis (DF) is a rare, locally invasive, non-metastasizing soft tissue proliferation derived from mesenchymal progenitor cells. The incidence of DF is 2 to 4 per million per year in the general population and typically affects adults between the ages of 35 - 40. Desmoid-type fibromatosis can either be sporadic or associated with mutation in the adenomatous polyposis coli gene. Trauma, surgery, pregnancy, and oral contraceptives have been identified as risk factors for the development of desmoid-type fibromatosis. MRI is the standard for image characterization, and CT image-guided core needle biopsy for diagnosis. "Wait and see" is the current management recommendation, and studies of y-secretase inhibitors and tyrosine kinase inhibitors have shown promise in the treatment of desmoid-type fibromatosis. This report presents a case of rare right shoulder desmoid type fibromatosis in a 48-year-old male that was missed on an initial workup including EMG/NCS and shoulder MRI, and demonstrates the importance of revisiting the diagnostic process if a former workup has yielded an unclear clinical picture.

\section{INTRODUCTION}

Desmoid-type fibromatosis (DF) is a rare, locally invasive, non-metastasizing soft tissue proliferation derived from mesenchymal progenitor cells [1]. The incidence of DF is 2 to 4 per million per year in the general population, and is reportedly 1000 -fold higher in patients with a mutated adenomatous polyposis coli (APC) gene resulting in familial adenomatous polyposis (FAP) [2]. DF spreads across planes and muscles and infiltrates surrounding structures. Trauma, surgery, pregnancy, and oral contraceptives have 
been identified as risk factors for the development of DF $[2,3]$. The most common primary sites are the abdominal wall, limbs, girdles, and mesenteric area. DF typically affects young adults between the ages of 35 - 40 and results in pain, functional impairment, and, rarely, can be life-threatening. Patients affected by DF face long-lasting morbidity, and require supportive care [4]. There are two types of DF: sporadic and those associated with germline APC mutations. $85 \%-90 \%$ of DF is sporadic and is associated with somatic CTNNB1 mutations. The remaining 10\% - 15\% of DF is associated with germline mutations of APC which provide different syndromic associations including Familial Adenomatous Polyposis and Gardner syndrome. CTNNB1 or APC mutations are mutually exclusive; therefore, a "wild-type" without CTNNB1 or APC mutations does not exist or is misdiagnosed. The natural course of DF is unpredictable. MRI is the most appropriate imaging method to characterize initial extension of DF, and CT image-guided core-needle biopsy is required to formally diagnose. Up to one-third are misdiagnosed and a second opinion by an expert pathologist is recommended [5]. Studies suggest a high recurrence rate after surgery [6]; therefore, large en-bloc surgery is not recommended as cornerstone treatment, and "wait-and-see" is the current management recommendation. There are studies demonstrating effective response to y-secretase inhibitors and tyrosine kinase inhibitors [7]; however, more studies are needed to confirm their role as first line. DF of the shoulder accounts for $17 \%$ of the extra-abdominal types, and few cases have been described over the past decade [8]. The diagnosis of shoulder DF is challenging to make due to its low incidence, variable clinical course, and nonspecific symptoms and is unlikely to be on most differential diagnoses for shoulder weakness and atrophy. We present a rare case of right shoulder DF in a 48-year-old male with a history of rotator cuff surgery that was missed on an initial workup including an electromyography and nerve conduction study (EMG/NCS) and shoulder MRI, and was identified nearly one year later after an abnormal limited EMG prompted ordering of a brachial plexus MRI that was converted to chest MRI. This case demonstrates the importance of revisiting the diagnostic process if a former workup has yielded an unclear clinical picture.

\section{CASE PRESENTATION}

A 48-year-old male with no past medical history and a surgical history of uncomplicated right shoulder rotator cuff repair presented to us with ten months of right neck spasms and winging and snapping of his right scapula. One year after his rotator cuff surgery, the patient noticed paracervical and right scapular pain with repetitive ball throwing and weight lifting. The patient had previously been diagnosed with dorsal scapular nerve entrapment. A past EMG/NCS revealed chronic neurogenic changes in the right biceps and right deltoid muscles which were thought to be due to chronic C5-C6 radiculopathy. A past shoulder MRI for chronic shoulder pain was read as no evidence of disease, and a past MRI C-Spine showed slight neural foraminal narrowing at $\mathrm{C} 4-\mathrm{C} 5$ and mild disc bulge in $\mathrm{C} 3-\mathrm{C} 4, \mathrm{C} 5-\mathrm{C} 6$, and $\mathrm{C} 7-\mathrm{T} 1$. His daily pain score was 6-7/10. $1000 \mathrm{mg}$ acetaminophen Q6H PRN, $800 \mathrm{mg}$ ibuprofen Q8H PRN, $440 \mathrm{mg}$ naproxen sodium BID PRN, physical therapy, acupuncture, chiropractic adjustments, deep needling, tissue massage, and a scalene injection had not provided relief. The family history included cervical dystonia in the patient's father.

On our physical examination, there was audible clicking and clunking of the scapulothoracic joint that improved with glenohumeral joint stabilization. There was tenderness along the cervical paraspinals. Tenderness was also present along the anterior shoulder at the pectoralis minor attachment site. There was a good range of motion of both shoulders and cervical spine. On neurological examination, there was noticeable atrophy of the pectoralis complex on the right as compared to the left. There was weakness of the right deltoid and biceps as compared to the left. Right biceps reflex was absent. A limited EMG was performed which revealed atrophy of the pectoralis, infraspinatus, and supraspinatus muscles.

A C6 selective nerve root block was performed that did not provide relief for the patient, and a brachial plexus MRI was ordered. The brachial plexus MRI was converted to a chest MRI with and without contrast. The chest MRI with and without contrast showed a $5.3 \times 3.8 \times 5.6 \mathrm{~cm}$ enhancing soft tissue mass medial and contiguous with the scapula and coracoid process (Figure 1). 


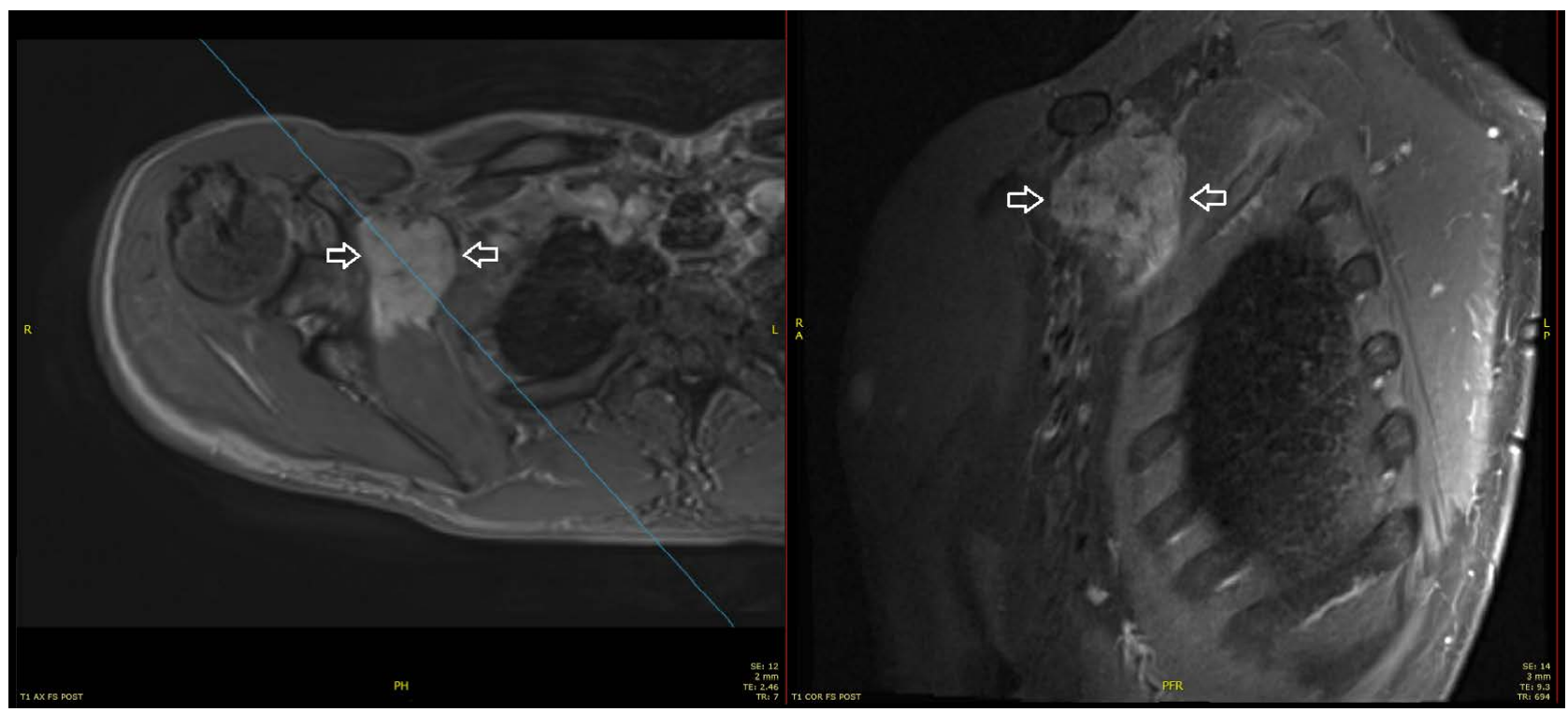

Figure 1. $5.3 \times 3.8 \times 5.6 \mathrm{~cm}$ enhancing soft tissue mass medial and contiguous with the scapula and coracoid process on $\mathrm{T} 1$ weighted axial (right) and coronal (left) chest imaging post contrast on Siemens 3 Tesla MRI.

The patient underwent CT guided bone biopsy which was consistent with desmoid tumor with beta-catenin IHC positive in the nuclei on the surgical pathology report. The patient decided to follow up care with an outside hospital medical oncology team, and was started on sorafenib $400 \mathrm{mg}$ daily with orders for follow-up MRI in 3 months. At the patient's most recent visit with his outside medical oncology team, he had clinically improved with reduction of shoulder pain and improved range of motion and the mass had reduced to $4.9 \times 3.8 \times 4.8 \mathrm{~cm}$ in size. He had been experiencing some diarrhea, hand-foot discomfort, and erectile dysfunction, all of which are potential side effects of sorafenib. His medical oncology team decreased the sorafenib dose to $200 \mathrm{mg}$ daily to reduce side effects and plans to follow up with reimaging by MRI in 6 months or with a change in symptoms.

\section{DISCUSSION}

$\mathrm{DF}$ is a rare, locally invasive, non-metastasizing soft tissue derived from mesenchymal progenitor cells. DF localization can be intra or extra abdominal, with abdominal wall, limbs, girdles, and mesenteric area being the most common sites affected. DF can either be sporadic or associated with germline APC mutations. Sporadic desmoid tumors comprise $85 \%-90 \%$ of cases and are associated with CTNNB1 mutations. The $10 \%-15 \%$ of DF associated with germline mutations of APC provide syndromic associations including Familial Adenomatous Polyposis Syndrome and Gardner Syndrome. MRI is the standard for imaging characterization of DF with CT image-guided core-needle biopsy for formal diagnosis. Reliable and validated predictive and prognostic factors have not yet been identified [2]; however there are multiple reports of spontaneous stabilization $[9,10]$, and one retrospective study showed young age $<40$ years and tumor recurrence to be associated with longer time to spontaneous stabilization [11]. DF of the shoulder accounts for $17 \%$ of the extra-abdominal type, and few cases have been described over the past decade [8].

Our patient had risk factors for DF, including a history of surgery and a relatively younger age. The diagnosis of DF was made ten months after the patient's symptoms began and after an initial workup was performed with misdiagnosis. We reviewed the past shoulder MRI, and we were able to visualize the tumor on the shoulder MRI. The tumor was proximal to the shoulder complex, and was easy to miss if not looking for it specifically. The tumor was not visible on the past MRI C-spine. Unfortunately, the delay in diagnosis was extended by nearly a year because of the miss on the first workup. On our limited EMG, 
atrophy of the pectoralis, supraspinatus, and infraspinatus were inconsistent with typical C6 radiculopathy which prompted ordering of the brachial plexus MRI. The tumor was fully appreciated when the brachial plexus MRI was converted to a chest MRI with and without contrast, and diagnosis of DF was confirmed with CT guided biopsy. Factors contributing to the delay in diagnosis include the low incidence of DF, variable clinical course, nonspecific symptoms, difficulty visualizing the mass on the past shoulder MRI, and limitation of EMG/NCS in differentiating between C5/C6 radiculopathy and a mass compressing the peripheral nerves or the brachial plexus in a C5/C6 distribution. While unlikely to be included on most differential diagnoses for shoulder weakness and atrophy, one should consider shoulder DF or other soft tissue masses in a patient with treatment-resistant progressive radiculopathy-like symptoms. It is important to revisit the diagnostic process if a patient is reporting worsening of symptoms or if a former workup has resulted in an unclear clinical picture.

\section{CONCLUSION}

Diagnosis of shoulder DF is challenging due to its nonspecific clinical presentation and low incidence. Shoulder DF may be considered a differential diagnosis for shoulder atrophy and weakness when a patient presents with treatment-resistant progressive radiculopathy-like symptoms. It is important to revisit the diagnostic process if a patient is reporting worsening of symptoms or if a former workup has resulted in an unclear clinical picture.

\section{CONFLICTS OF INTEREST}

The authors declare no conflicts of interest regarding the publication of this paper.

\section{REFERENCES}

1. Wu, C., Amini-Nik, S., Nadesan, P., Stanford, W.L. and Alman, B.A. (2010) Aggressive Fibromatosis (Desmoid Tumor) Is Derived from Mesenchymal Progenitor Cells. Cancer Research, 70, 7690-7698.

https://doi.org/10.1158/0008-5472.CAN-10-1656

2. Skubitz, K.M. (2017) Biology and Treatment of Aggressive Fibromatosis or Desmoid Tumor. Mayo Clinic Proceedings, 92, 947-964. https://doi.org/10.1016/j.mayocp.2017.02.012

3. Bertario, L., Russo, A., Sala, P., Eboli, M., Giarola, M., D’amico, F., et al. (2001) Genotype and Phenotype Factors as Determinants of Desmoid Tumors in Patients with Familial Adenomatous Polyposis. International Journal of Cancer, 95, 102-107. https://doi.org/10.1002/1097-0215(20010320)95:2<102::AID-IJC1018>3.0.CO;2-8

4. Penel, N., Chibon, F. and Salas, S. (2017) Adult Desmoid Tumors: Biology, Management and Ongoing Trials. Current Opinion in Oncology, 29, 268-274. https://doi.org/10.1097/CCO.0000000000000374

5. Penel, N., Coindre, J.M., Bonvalot, S., Italiano, A., Neuville, A., et al. (2016) Management of Desmoid Tumours: A Nationwide Survey of Labelled Reference Centre Networks in France. European Journal of Cancer, 58, 90-96. https://doi.org/10.1016/j.ejca.2016.02.008

6. Crago, A.M., Denton, B., Salas, S., Dufresne, A., Mezhir, J., et al. (2013) A Prognostic Nomogram for Prediction of Recurrence in Desmoid Fibromatosis. Annals of Surgery, 258, 347-353.

https://doi.org/10.1097/SLA.0b013e31828c8a30

7. Wang, Z., Wu, J., Tian, X. and Hao, C. (2019) Targeted Therapy of Desmoid-Type Fibromatosis: Mechanism, Current Situation, and Future Prospects. Frontiers in Medicine, 13, 427-437.

https://doi.org/10.1007/s11684-018-0672-6

8. Tang, F., Min, L., Yin, R., Zhang, W., Zhou, Y., et al. (2015) Large Desmoid-Type Fibromatosis of the Shoulder Girdle: Operative Approach Selection and Clinic Outcome. International Orthopaedics, 39, 363-369. https://doi.org/10.1007/s00264-014-2660-z 
9. Stoeckle, E., Coindre, J.M., Longy, M., Binh, M.B., Kantor, G., et al. (2009) A Critical Analysis of Treatment Strategies in Desmoid Tumours: A Review of a Series of 106 Cases. European Journal of Surgical Oncology, 35, 129-134. https://doi.org/10.1016/j.ejso.2008.06.1495

10. Bonvalot, S., Eldweny, H., Haddad, V., Rimareix, F., Missenard, G., et al. (2008) Extra-Abdominal Primary Fibromatosis: Aggressive Management Could Be Avoided in a Subgroup of Patients. European Journal of Surgical Oncology, 34, 462-468. https://doi.org/10.1016/j.ejso.2007.06.006

11. Kim, Y., Rosario, M.S., Cho, H.S. and Han, I. (2020) Factors Associated with Disease Stabilization of Desmoid-Type Fibromatosis. Clinics in Orthopedic Surgery, 12, 113-119. https://doi.org/10.4055/cios.2020.12.1.113 